docking programs and ligand-based design.

If the structure of the target protein's active site is known, the company's SiteExplorer can predict potential drugbinding sites, and can evaluate interactions between these sites and the drug candidates. If the structure is unknown, then its Quasi2 software will produce a virtual protein based on molecular features known to be important in binding in other targets. Drugs can then be designed against the model.

De Novo also offers software to aid the design of chemical probes used in target validation. The SkelGen suite of programs can then use these data to generate new chemical structures optimized for interaction with a target's active site.

The company is collaborating with GeneFormatics of San Diego, California, in a programme focused on inhibitors of the M10 family of matrix metalloproteinases, enzymes that are involved in cancer and inflammatory disorders. GeneFormatics is using proteomics to identify the target enzymes and characterize their active sites, while De Novo is running docking models and virtual screens of small molecules against the proteins.

Software that can model drug-receptor interactions is available from a number of companies including Tripos of St Louis, Missouri; Accelrys in San Diego, California; and Metaphorics in Mission Viejo, California. In addition, some software is available free to researchers at non-profit organizations, such as AutoDock 3 made by the Scripps Research Institute in La Jolla, California, and GOLD from the Cambridge Crystallographic Data Centre, UK. Molsoft in La Jolla, California, which makes the ICM molecular modelling software, last month released an ICM browser for the Apple Macintosh.

The Accelrys suite of structural homology programs identifies the possible function, fold family and threedimensional structure of target proteins by comparing them with sequences and structural homologues of known function. Once the protein's structure is determined, functional information can be gleaned using different modules within Accelrys's Insight II program, which supports a number of processes including X-ray crystallography, nuclear-magneticresonance studies and protein engineering.

Target Engine from LION Bioscience in Heidelberg, Germany, aids target selection by offering the ability to analyse gene sequence and expression data, find homologous structures, map potential functional features onto protein structure, view related gene annotation and protein pathway information and use text mining to find functional relationships.

In biotherapeutics, proteins themselves are developed as active drugs. One software suite designed to help optimize protein function is Protein Design Automation (PDA) produced by Xencor. "We don't

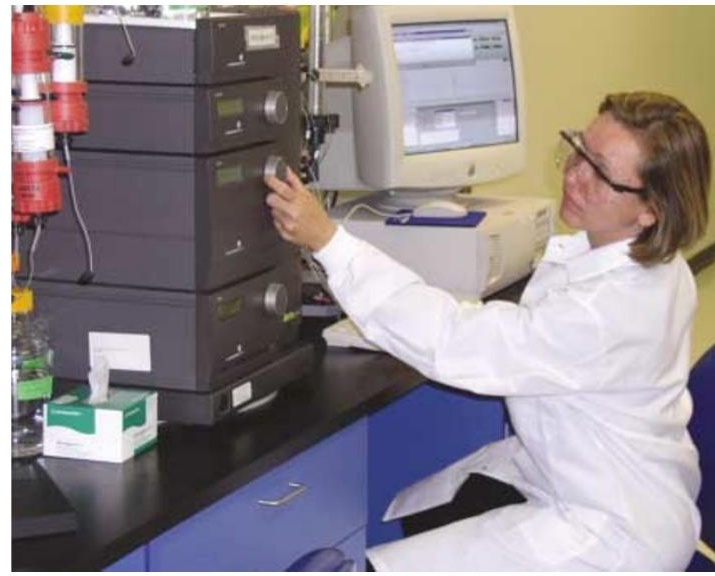

Purifying proteins for functional assessment at Xencor

screen DNA sequences," says Szymkowski. "More specifically, PDA computationally screens massive numbers of amino-acid changes in a known protein structure." It then derives functional information from the three-dimensional protein structure and designs novel features into the protein to optimize its function.

\section{Sense reversal}

Another route to target validation hinges on disrupting gene expression to reduce the amount of the corresponding protein, and so identify the physiological role of the target. Examples of this technique include gene knockouts, antisense

\title{
THE SILENT TREATMENT
}

RNA interference (RNAi) is a selective method of silencing protein expression at the post-transcriptional level. Double-stranded RNA specific to the gene to be silenced is introduced into the cell, where it is processed into short single-stranded RNA fragments. Antisense strands, complementary to the fragments, bind to the target RNA and prompt enzymes to disable it. The effect is to destroy all the target messenger RNA, effectively halting production of the protein.

Genetica, a start-up company in Cambridge, Massachusetts, co-founded by Gregory Hannon at Cold Spring Harbor Laboratory, New York, is developing RNAi technology for high-throughput target validation in mammalian cells. Hannon and his colleagues in New York have done much of the recent work in the stable RNAi suppression of gene expression in mammalian cells.

Scientists at Bristol-Myers Squibb, based in Princeton, New Jersey, are trying to create RNAi reagents for the entire proteome, to allow analysis of all expressed genes. But there are still problems with the method, says Pam Carroll, senior research investigator in applied genomics at the company. "Most researchers use synthetic RNA double-stranded oligos that are expensive and there is still variability in response. Nonetheless, it has been an amazing year for RNA interference as

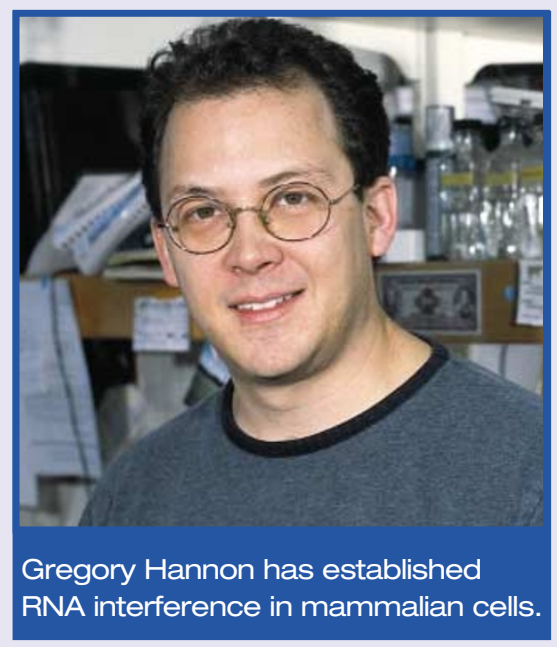

a mammalian target validation technology," she says. For example, only recently has RNAi been successfully used in mammalian model systems.

Another exciting development in RNAi is the use of small interfering RNA (siRNA) compounds. These are "efficient, specific and relatively non-toxic", says Dmitry Samarsky, manager of technology development at functional-genomics firm Sequitur in Natick, Massachusetts. But he notes that getting these compounds into cells is still a challenge. For researchers interested in a convenient approach to RNAi, companies such as OligoEngine in Seattle, Washington, and Ambion in Austin, Texas, offer siRNAi kits and expression vectors, as well as custom siRNA synthesis. Custom siRNAs are also now supplied by many RNA companies such as Dharmacon in Lafayette, Colorado; Proligo in Hamburg, Germany; and QIAGEN in Venlo, the Netherlands.

The biotech firm Benitec, in St Lucia, Australia, has circumvented the difficulties of introducing double-stranded RNA into cells by developing a DNA template that produces double-stranded hairpin-loop molecules within the cell, which trigger RNAi. Last September, Benitec announced that it had made the first transgenic mice in which targeted endogenous genes were suppressed using RNAi. 